\title{
A methoxy derivative of resveratrol analogue selectively induced activation of the mitochondrial apoptotic pathway in transformed
} fibroblasts

\author{
A Gosslau ${ }^{1,2}$, M Chen ${ }^{1,2}$, Ci-T Ho ${ }^{1,2}$ and KY Chen ${ }^{*, 1,2}$ \\ 'Department of Chemistry and Chemical Biology, Center for Advanced Food Technology, Rutgers, The State University of New Jersey, Piscataway, NJ \\ 08854-8087, USA; ${ }^{2}$ New Jersey Cancer Institute, New Brunswick, NJ 0890 I, USA
}

Resveratrol (R-3), a trihydroxy trans-stilbene from grape, inhibits multistage carcinogenesis in animal models. A resveratrol derivative 3,4,5,4'-tetrahydroxystilbene (R-4) exhibits potent growth inhibitory effect against transformed human cells. Here we report that 3,4,5,4'-tetramethoxystilbene (MR-4), converted from R-4, was more potent against cancer cell lines (WI38VA, IMR-90SV, HeLa, LNCaP, HT-29, and HepG2), but had almost no inhibitory effect on the growth of normal cells (WI38, IMR-90, BJ-T) at the concentrations tested. The $\mathrm{IC}_{50}$ value of MR-4 on the growth inhibition of transformed WI38VA human cells was $0.5 \mu \mathrm{M}$, as compared to the value of greater than $50 \mu \mathrm{M}$ for the normal WI38 cells. Resveratrol, however, did not exhibit such clear differential effect and the IC 50 value of R-3 for WI38VA cells was about $50 \mu \mathrm{M}$. The growth inhibitory effect of MR-4 correlated with the induction of apoptosis in the transformed cells. When normal WI38 cells and transformed WI38VA cells were compared, MR-4 induced increases of the $\mathrm{Bax} / \mathrm{Bcl}-2$ mRNA ratio, p53 and Bax protein level, activation of caspases, and DNA fragmentation in transformed, but not in normal cells. Further analysis revealed that MR-4 caused a rapid appearance of perinuclear aggregation of mitochondria in WI38VA but not in WI38 cells, suggesting that the mitochondria could serve as an early target of MR-4. R-3 also induced apoptosis and mitochondrial clustering but only at a much higher concentration, close to $500 \mu \mathrm{M}$. Taken together, the specific activation of the mitochondria-mediated apoptotic pathway could be a major reason for the striking differential growth inhibitory effect of MR-4.

British Journal of Cancer (2005) 92, 513-521. doi: I 0.1038/sj.bjc.6602300 www.bjcancer.com

Published online 25 January 2005

(C) 2005 Cancer Research UK

Keywords: resveratrol; chemoprevention; apoptosis; mitochondria

Resveratrol (3,5,4'-trihydroxy-trans-stilbene), a phytoalexin present in grapes, peanuts, and pines, has antioxidant and antiinflammatory activities (Jeandet et al, 1991), and is the active ingredient in Leguminosae that inhibits cellular events associated with tumour initiation, promotion, and progression in mouse skin cancer model (Jang et al, 1997). In vitro, resveratrol inhibits the growth of various human tumour cells, including oral squamous carcinoma (Elattar and Virji, 1999), promyelocytic leukemia (Surh et al, 1999), human breast cancer cells (Lu and Serrero, 1999), prostate cancer cells (Hsieh and $\mathrm{Wu}, 1999$; Narayanan et al, 2003), oesophageal carcinoma cells (Zhou et al, 2003), pancreatic cancer cells (Ding and Adrian, 2002), and monocytic leukaemia cell (Tsan et al, 2000). The potential of resveratrol as a cancer chemoprevention reagent has been extensively reviewed (Gusman et al, 2001; Bhat and Pezzuto, 2002; Savouret and Quesne, 2002; Aziz et al, 2003). The possible role of resveratrol, a phytoestrogen, in cardiovascular protection has also been discussed recently (Hung et al, 2001; Wu et al, 2001). Nevertheless, there are two concerns

*Correspondence: Dr KY Chen, Department of Chemistry and Chemical Biology, Rutgers University, 610 Taylor Road, Piscataway, NJ 08854-8087, USA; E-mail: kychen@rutchem.rutgers.edu

Revised II October 2004; accepted 22 October 2004; published online 25 January 2005 with regard to the use of resveratrol as chemoprevention agent. First, most of the reported studies did not use paired normal and cancer cells to compare the effects of resveratrol. For effective chemoprevention, it is desirable that the candidate compound demonstrates clear differential growth inhibitory effect against cancer cells. Second, the $\mathrm{IC}_{50}$ of growth inhibition for resveratrol, which varied between 40 and $200 \mu \mathrm{m}$ as reported in the literature for various cell types, is relatively high. To address these concerns, we have used a matched pair of normal and transformed human fibroblasts to compare the antiproliferative activity of resveratrol and other analogues derived from resveratrol (Lu et al, 2001). We have observed a selective induction of apoptosis in cancer cells by R-4 (3,4,5,4'-tetrahydroxystilbene) (Lu et al, 2001).

Apoptosis can be considered as a proactive self-defense mechanism of a living organism to weed out dysfunctional cells such as the precursors of metastatic cancer cells without creating secondary oxidative stress due to inflammation (Davies, 2000). Aberration of apoptotic pathways leads to cancer and other diseases (Igney and Krammer, 2002; Reed, 2003). Apoptosis is defined by a set of energy-dependent changes including chromatin condensation, DNA fragmentation, membrane blebbing, and cell shrinkage. These events lead ultimately to cell death (Green and Reed, 1998; Hengartner, 2000; Reed, 2002; vanGurp et al, 2003). There exist two major pathways that initiate apoptosis: the 
extrinsic (receptor-mediated) and intrinsic (mitochondriamediated) pathway, which can crosstalk to each other (Green and Reed, 1998; Hengartner, 2000; Reed, 2002; vanGurp et al, 2003; Gosslau and Chen, 2004). The extrinsic pathway is triggered by the binding of ligands (e.g. TNF) to their cell death receptors (TNFreceptor) located on the plasma membrane (Strater and Moller, 2000; Peter and Krammer, 2003). Activation is triggered by receptor trimerisation and recruitment of associated proteins, which result in activation of procaspase- 8 and caspase- 3 activation (Strater and Moller, 2000; Peter and Krammer, 2003; Gosslau and Chen, 2004).

The crucial step in the mitochondria-mediated apoptosis is the change of mitochondrial membrane permeability, and collapse of membrane potential (Green and Reed, 1998; Hengartner, 2000; Reed, 2002; vanGurp et al, 2003; Gosslau and Chen, 2004). The change can be induced by (i) an opening of the permeability transition pore (PTP); (ii) an increase of the $\mathrm{Bax} / \mathrm{Bcl}-2$ ratio; or (iii) reactive oxygen species (ROS)-induced damage of mitochondrial membrane (Green and Reed, 1998; Hengartner, 2000; Reed, 2002; vanGurp et al, 2003; Gosslau and Chen, 2004). The change in membrane permeability causes mitochondrial swelling, rupture of the outer membrane, and release of proapoptotic factors such as cytochrome $c$, pro-caspases, Apaf-1, and AIF, from the intermembranous space, which leads to the formation of a supramolecular apoptosome complex that turns on the caspase cascade (Green and Reed, 1998; vanGurp et al, 2003). Increase of the Bax/Bcl-2 ratio is proapoptotic by inducing permeabilisation of mitochondria. Caspase-3 serves as the 'central executioner of apoptosis' since it is activated by the extrinsic as well as intrinsic pathway (Susin et al, 1997). Caspase-3 activates other caspases, cleaves cytoskeletal proteins (e.g. fodrin and gelsolin), or activates the caspaseactivated DNase (CAD), which is involved in fragmentation of nuclear DNA. The characteristic cleavage of DNA into nucleosomal fragments has been regarded as a hallmark of apoptosis (Compton, 1992).

Recently, we reported that among eight resveratrol derivatives, R-4 (3,4,5,4'-tetrahydroxy-trans-stilbene) inhibited the growth of transformed cells, but had little effect on the growth of the normal cells (Lu et al, 2001). We now report that MR-4 (3,4,5,4'tetramethoxy-trans-stilbene), the methoxy derivative of R-4, exhibits a striking differential growth inhibitory effect in several different cancer cell lines. Noteworthy, MR-4 selectively eliminated cancer cells by inducing apoptosis and the apoptotic signalling may be triggered at mitochondria, which show very early perinuclear aggregation after MR-4 treatment. Taken together, our study suggests that resveratrol may serve as a useful chemical platform for generating more potent anticancer compounds targeting at mitochondria-mediated apoptotic pathway.

\section{MATERIALS AND METHODS}

\section{Materials and chemicals}

Dulbecco's modified Eagle's medium (DMEM) and fetal bovine serum (FBS) were obtained from Gibco BRL (Gaithersburg, MD, USA). $\left[\alpha-{ }^{33} \mathrm{P}\right] \mathrm{dCTP}$ was purchased from Perkin Elmer (Boston, MA, USA). Oligo-dT, poly-dA, dNTPs, Superscript ${ }^{\mathrm{TM}}$ II reverse transcriptase, Cot-1 DNA, MicroHyb hybridisation solution were purchased from Invitrogen Life Technologies (Baltimore, MD, USA). Anti-p53 antibody conjugated to horseradish peroxidase (polyclonal BMG-1B1) was purchased from Roche (Indianapolis, IN, USA), and antibodies for Bax, Bcl-2, and $\beta$-actin were from Santa Cruz iotechnology (Santa Cruz, CA, USA). Secondary antimouse (NIF 825) and anti-rabbit (NIF 824) conjugated to horseradish peroxidase were from Amersham Pharmacia (Piscataway, NJ, USA). Other chemicals were purchased from Sigma (St Louis, MO, USA).
Preparation of resveratrol (R-3) and and 3,4, $4^{\prime}, 5^{\prime}$ tetramethoxystilbene (MR-4).

Polyhydroxystilbenes and polymethoxystilbenes are designated as $\mathrm{R}$ or MR followed by a number indicating the number of hydroxyl or methoxyl groups (Lu et al, 2001). Whereas the natural occurring R-3 (3,5,4'-trihydroxy-trans-stilbene) contains only two hydroxyl groups in the first benzene ring, its analogues R-4 $\left(3,4,5,4^{\prime}-\right.$ tetrahydroxy-trans-stilbene) and MR-4 (3,4,5,4'-tetramethoxytrans-stilbene) contain, respectively, three hydroxyl and methoxyl groups. The second benzene ring is either hydroxylated (R-3 and $\mathrm{R}-4$ ) or methoxylated (MR-4). The synthesis of resveratrol using 4methoxybenzyl alcohol and 3,5-dimethoxy-benzaldehyde as the starting materials has been described (Bachelor et al, 1970; Drewes and Fletcher, 1974). Similar strategy was employed to prepare MR4. The identity and purity of each of these compounds has been confirmed by thin layer chromatography, NMR and GC-mass spectroscopy.

\section{Cell culture and treatment}

Different human normal cells including WI38 (normal lung fibroblasts), BJ-T (normal foreskin fibroblasts transfected with telomerase), and IMR-90 (normal fibroblasts) or human tumour cell lines including WI38VA (transformed lung fibroblasts), IMR90SV (transformed fibroblast), HeLa (cervix cancer), LNCaP (prostate cancer), HT-29 (colon cancer), and HepG2 (hepatoma cells) were obtained from the National Institute on Aging Repository (Coriell Institute for Medical Research, Camden, NJ, USA). Cells were cultured in Dulbecco's modified Eagles medium (DMEM) supplemented with $10 \% \mathrm{FBS}$ at $37^{\circ} \mathrm{C}$ in a humidified, $10 \%$ $\mathrm{CO}_{2}$ atmosphere. Cells were subcultured in culture flasks (Falcon, Becton-Dickinson, Franklin Lakes, NJ, USA) and passaged every 3 days. Before experiments, cells were seeded either in $88,35-\mathrm{mm}$ culture dishes or in 24-well plates (Falcon, Becton-Dickinson, Franklin Lakes, NJ, USA) as indicated for the different assays to establish a confluent monolayer for untreated control cells at the end of the experiment. Different stock solutions of R-3 or MR-4 in DMSO were applied to the medium.

\section{Cellular proliferation assays and morphological analysis}

Cell proliferation was measured by the MTT (344,5-dimethylthiazol-2-yl)-2,5-diphenyl-tetrazolium-bromide) method, crystal violet staining, or by cell counting. For the MTT- and crystal violet assay, cells were treated in 24-well plates for 5 days, whereas for the counting cell numbers were determined at designated time points. The MTT-assay, based on the conversion of the tetrazolium salt MTT to blue formazan by mitochondrial dehydrogenase, measures mitochondrial damage (Berg et al, 1990). Owingto the concern that MTT assay may yield false-positive results for certain cell types when treated with flavonoids or polyphenols (Bernhard et al, 2003), we also included the crystal violet dye staining assay in this study (Lu et al, 2001). Colour development was documented by a scanner (UMAX, Astra 2200). For the counting method cells were analysed by phase contrast light microscopy and documented using a digital camera system (MDS 120, Kodak, Emeryville, CA, USA). For morphological analysis, cells were treated in $35-\mathrm{mm}$ cell culture dishes for 5 days. Morphological analysis was performed by phase contrast light microscopy and documented by the digital camera system (MDS 120, Kodak, Emeryville, CA, USA).

\section{DNA fragmentation assay}

After treatment in $60-\mathrm{mm}$ culture dishes, cells were harvested and resuspended in $500 \mu$ l of lysis buffer $\left(0.5 \%\left(\mathrm{wv}^{-1}\right)\right.$ sodium lauryl sarkosinate $+10 \mathrm{~mm}$ EDTA $+0.5 \mathrm{mg} \mathrm{m}^{-1}$ proteinase $\mathrm{K}+0.1 \mathrm{mg} \mathrm{ml}^{-1}$ RNase $\mathrm{A}$ in $50 \mathrm{~mm}$ Tris-Base, $\mathrm{pH} 8.0$ ). After an 
incubation for $40 \mathrm{~min}$ at $37^{\circ} \mathrm{C}$, proteins were precipitated in $1 \mathrm{M}$ $\mathrm{NaCl}$ at $4^{\circ} \mathrm{C}$ for $1 \mathrm{~h}$, followed by a centrifugation $(10000 \times \mathrm{g}$ for $10 \mathrm{~min})$. DNA from the supernatant was then extracted with a phenol/chloroform/isopropanol mixture (25:24:1, pH 8.0), precipitated by ethanol (70\%), and dried in a Speed Vac. For separation, DNA pellets were resuspended in water $(20 \mu \mathrm{l})$ and $10 \mu \mathrm{l}$ of DNA laddering loading buffer (1\% low-gelling temperature agarose $\left(\mathrm{w} \mathrm{v}^{-1}\right)+0.25 \%$ bromphenol blue $\left(\mathrm{w} \mathrm{v}^{-1}\right)+40 \%$ sucrose in $10 \mathrm{ml}$ EDTA, $\mathrm{pH} 8.0$ ), which was heated to $100^{\circ} \mathrm{C}$, previously. After loading onto a $2 \%$ agarose gel, the samples were solidified for $1 \mathrm{~h}$. Thereafter, DNA samples were separated by electrophoresis, stained by ethidium bromide and visualised under UV illumination.

\section{Caspase activation assay}

The effect of MR-4 on caspase activation was analysed by CaspACE ${ }^{\mathrm{TM}}$ FITC-VAD-FMK in situ marker (Promega, Madison, WI, USA), which is a fluoroisothiocyanate (FITC) conjugate of the cell permeable caspase inhibitor VAD-FMK. The structure allows delivery of the inhibitor into the cells where it binds to activated caspases. Cells were seeded on coverslips 2 days before experiment. After treatment, cells on coverslips were transferred onto a $35-\mathrm{mm}$ dish containing $1 \mathrm{ml}$ of medium. Then, $2 \mu \mathrm{l}$ of CaspACEsolution were applied to the medium to achieve a final concentration of $10 \mu \mathrm{M}$. After an incubation for $20 \mathrm{~min}$, cells were analysed by fluorescent microscopy using an excitation wavelength of $480 \mathrm{~nm}$.

\section{Reverse transcription-polymerase chain reaction (RT - PCR)}

After treatment, cells were harvested at indicated times and the total RNA was prepared using RNeasy ${ }^{\mathrm{TM}}$ Total RNA Kit (Qiagen, Chatsworth, CA, USA). Then, total RNA $(1 \mu \mathrm{g})$ was reverse transcribed into cDNA by incubating with SuperScriptTM RNase $\mathrm{H}$ reverse transcriptase (Invitrogen Life Technologies, Baltimore, $\mathrm{MD}$, USA) using oligo(dT) $)_{12-18}$ as primer (Invitrogen Life Technologies, Baltimore, MD, USA). For PCR amplification, gene specific primers, both sense and antisense, were used. The sequences of these primers were:

(Bax sense) $=5^{\prime}$-CTg ACA TgT TTT CTg ACg gC-3';

(Bax antisense $)=5^{\prime}$-TCA gCC CAT CTT CTT CCA gA-3';

$(\mathrm{Bcl}-2$ sense $)=5^{\prime}-\mathrm{ACT}$ TgT ggC CCA gAT Agg CAC CCA g - $3^{\prime}$;

(Bcl-2 antisense) $=5^{\prime}$-gCg ACT TCg CCg AgA TgT CCA gC- $3^{\prime}$;

(GAPDH sense) $=5^{\prime}-\mathrm{TgA}$ AgC TCg gAg TCA Acg gAT TTg- ${ }^{\prime}$;

$($ GAPDH antisense $)=5^{\prime}$-CAT gTg ggC CAT gAg gTC CAC CAC- $3^{\prime}$.

PCR conditions were chosen to ensure that the yield of the amplified product was linear with respect to the amount of input RNA. The expression of glyceraldehyde-3-phosphate dehydrogenase (GAPDH), a housekeeping gene, was used as an internal control. The PCR products were analysed by electrophoresis on a $1 \%$ agarose gel and visualised by ethidium bromide staining.

\section{SDS-PAGE and Western blot}

After treatment, cells were harvested, washed, heated, and sonicated in lysis buffer ( $5 \mathrm{~mm}$ Tris-base ( $\mathrm{pH}$ 6.8), 5\% 2mercaptoethanol, $3 \%$ sodiumdodecylsulfate (SDS), $10 \%$ glycerol). After centrifugation $\left(18000 \times g\right.$ for $10 \mathrm{~min}$ at $\left.20^{\circ} \mathrm{C}\right)$, equal amounts of protein were separated by SDS-PAGE (10\%) and transferred onto a nitrocellulose membrane for Western blot analysis. Blots were blocked with $1 \%$ skim milk and $0.2 \%$ Tween 20 in phosphatebuffered saline (PBST) for $0.5 \mathrm{~h}$ at room temperature. Primary antibodies used were: anti-p53 antibody conjugated to horseradish peroxidase (polyclonal BMG-1B1, Roche, Indianapolis, IN, USA), anti-Bax (rabbit polyclonal, sc-526), anti-Bcl-2 (mouse monoclonal, sc-509), and anti- $\beta$-actin (mouse monoclonal, sc-8432), all from Santa Cruz Biotechnology, Santa Cruz, CA, USA. Secondary anti-mouse (NIF 825) and anti-rabbit (NIF 824) conjugated to horseradish peroxidase were from Amersham Pharmacia (Piscataway, NJ, USA). Primary and secondary antibodies were incubated for $1 \mathrm{~h}$ followed by three washes in PBST. Immunocomplexes were detected with the ECL Plus Western blot detection kit (Amersham Pharmacia, Piscataway, NJ, USA).

\section{Rhodamine 123 fluorescence assay}

Morphology of mitochondria was analysed using the cationic fluorophore rhodamine 123 which specifically localises mitochondria in living cells. Due to the negative electric potential across the mitochondrial membrane rhodamine 123 transfers electrophoretically into the mitochondria (Johnson et al, 1980). Before experiments, cells were grown on glass coverslips in $35-\mathrm{mm}$ culture dishes for 2 days. After treatment, $4 \mu \mathrm{l}$ of rhodamine 123 stock solution ( $1.5 \mathrm{~mm}$ in medium containing $10 \%$ DMSO) were added to the dishes to achieve a final concentration of $1.5 \mu \mathrm{M}$ and incubated for $20 \mathrm{~min}$. Then, coverslips were washed twice with medium to remove extracellular rhodamine. Cells were analysed either by phase contrast microscopy to monitor cell morphology or by fluorescence microscopy to determine mitochondrial morphology. For fluorescence microscopy, excitation and emission wavelength were 480 or $525 \mathrm{~nm}$, respectively.

\section{RESULTS}

\section{The effect of MR-4 and R-3 on normal and transformed human fibroblasts}

We first compared the effects of MR-4 and R-3 on the growth of normal and transformed human fibroblasts using the MTTreduction assay (Figure 1A). We also employed the crystal violet staining assay to monitor cell proliferation (Figure 1B). We found that both methods yielded qualitatively similar results. Human fibroblast WI38 and the virally transformed WI38 (termed WI38VA) were used as a model for paired normal and cancer cells in this study. Both cell lines exhibit comparable growth rate with the doubling time about $24 \mathrm{~h}$. For WI38 cells, both MR-4 and R-3 showed little or no inhibitory effect up to $50-100 \mu \mathrm{M}$ as determined by the MTT- or crystal violet assay. For WI38VA cells, MR-4 completely inhibited the growth at $1-2 \mu \mathrm{M}$, whereas R-3 was much less potent, being effective only at concentrations greater than $50 \mu \mathrm{M}$. Thus, MR- 4 was about 100 -fold more potent than R-3 in inhibiting the growth of transformed WI38VA cells. In addition to the MTT- and crystal violet assay, we also quantified the effect of MR-4 on growth rates of WI38 and WI38VA cells by viable cell counting (Figure 1C). When exposed to 0.5 or $1 \mu \mathrm{M}$, the differential growth inhibitory effect was evident 3 days after treatment. In summary, all three proliferation assays demonstrate that MR-4 exhibited a clear differential growth inhibitory effect toward transformed human cells. Moreover, MR-4 was about 50- to 100fold more potent than R-3 in inhibiting the growth of transformed cells.

\section{Effects of MR-4 on other normal and cancerous human cells}

The differential growth inhibitory effect of MR-4 on transformed WI38VA cells prompted us to examine whether this finding also holds for other cancer cell types. Figure 2 shows the phase contrast micrographs of three normal, two virally transformed (WI38VA, IMR-90SV), and one cancer cell line (HeLa) in the presence of 1 or $10 \mu \mathrm{M}$ of MR-4. At both concentrations MR-4 inhibited the growth of transformed and cancer cells. MR- 4 also inhibited the growth of LNCaP prostate cancer cells, HT-29 colon cancer cells, and HepG2 hepatoma cells with the $\mathrm{IC}_{50}$ values in the range of $1-5 \mu \mathrm{M}$ (data 
A
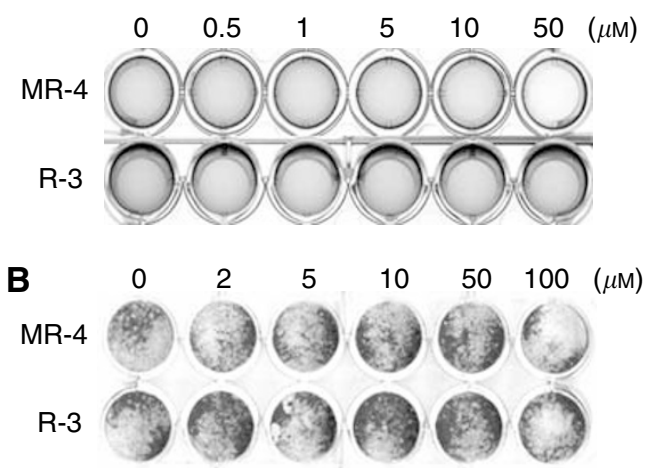

WI38VA
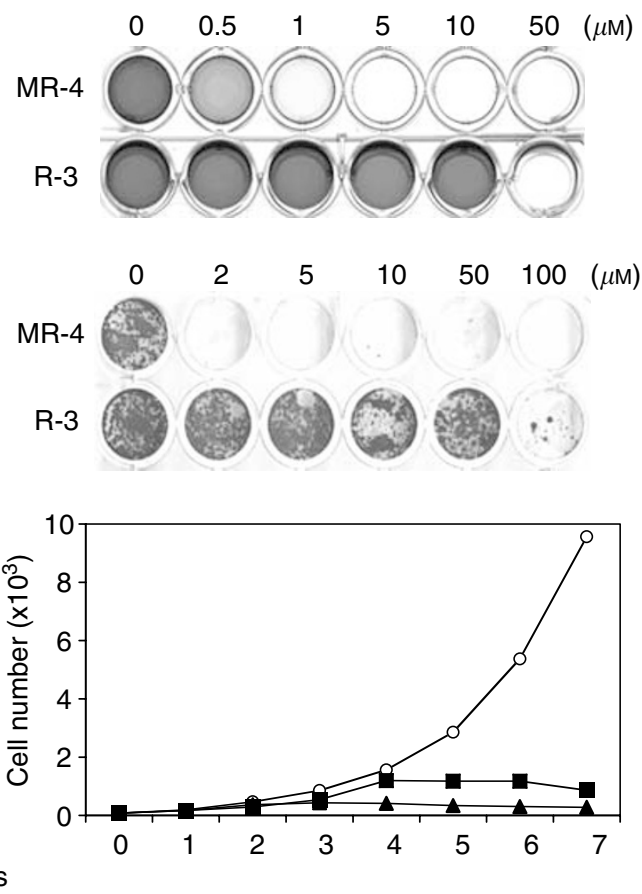

Figure I Growth inhibition induced by MR-4 and R-3 in normal and transformed fibroblasts. Normal (WI38; left panel) or transformed fibroblasts (WI38VA; right panel) were treated by different concentrations of MR-4. Then, cell growth was either analysed by $(\mathbf{A})$ the MTT-assay and (B) the crystal violet assay after 5 days or by $(\mathbf{C})$ cell counting after different time points as described in Material and Methods. For cell counting, cells were treated by $0.5 \mu \mathrm{M}$ (closed squares), I $\mu \mathrm{M}$ (closed triangles), or were left untreated as controls (open circles) and counted at indicated times. Results obtained by the MTT- or crystal violet assay were documented by a scanner $(\mathbf{A}, \mathbf{B})$ or expressed as cell numbers for the cell counting method $(\mathbf{C})$. Representative data of three independent experiments are shown.

not shown). On the other hand, MR-4 did not significantly suppress the growth of normal cells such as WI38, IMR90, and BJ-T. Although MR-4 did not affect the growth and viability of the normal cells, it did cause slight changes in cell morphology at high concentrations $(>10 \mu \mathrm{M})$. These results suggest that the differential growth inhibitory effect of MR-4 appeared to be general for transformed and cancerous cells.

\section{Effect of MR-4 on apoptosis in normal and transformed human fibroblasts}

Abundant literature evidence suggests that the antiproliferative effect of resveratrol is due to its ability to induce apoptosis (Gusman et al, 2001). It is therefore likely that the differential growth inhibitory effect of MR-4 may be due to selective induction of apoptosis in transformed cells, but not in normal cells. To examine this possibility, we first determined whether MR- 4 could elicit DNA laddering, a signature event of apoptotic process (Compton, 1992; Green and Reed, 1998; vanGurp et al, 2003). Figure $3 \mathrm{~A}$ shows an extensive DNA fragmentation in transformed WI38VA cells $48 \mathrm{~h}$ after MR-4 treatment. In contrast, DNA laddering was not observed in normal WI38 cells throughout the entire time course. Caspases are a family of aspartyl-specific cysteine proteases that play a key role in transmitting and executing a whole array of apoptotic signals (Budihardjo et al, 1999). We therefore also examined whether MR-4 had differential effects on global caspase activation. Figure $3 \mathrm{~B}$ shows that the activation of caspases occurred only in WI38VA cells following MR-4 treatment, but not in WI38 cells. These results indicate that MR-4 selectively induced apoptotic signalling pathways in transformed cells but not in normal cells.

\section{Effect of MR-4 on the expression of apoptosis-related genes in normal and transformed human fibroblasts}

Mitochondria-mediated apoptotic pathways involve modulation of the expression of genes such as p53, the key upstream player in apoptosis, and Bcl-2 family genes (Green and Reed, 1998; Sheikh and Fornace, 2000). The $\mathrm{Bax} / \mathrm{Bcl}-2$ ratio has been considered to be a rheostat of the apoptosis because it is related to the control of the permeability of mitochondrial membranes via oligomerisation mechanism (Korsmeyer et al, 1993; Korsmeyer, 1999). Induction of p53, both at mRNA and protein level, in cells treated with resveratrol is thought to be the major cause for apoptosis (Huang et al, 1999). To examine whether MR-4 affects p53 and Bcl-2 family genes, we performed RT-PCR and Western blot analysis in normal and transformed fibroblasts.

Figure $4 \mathrm{~A}$ shows that at the mRNA level, the ratio of Bax and Bcl-2 gene expression increased in WI38VA cells and the value reached to four-fold $6 \mathrm{~h}$ after the MR-4 treatment while the Bax/ Bcl-2 ratio in WI38 cells decreased after MR-4 treatment. When analysed at protein level, we observed a prominent increase of p53 by more than 10-fold in WI38VA cells within $4 \mathrm{~h}$ (Figure 4B). Moreover, we noticed that although the Bcl-2 level in WI38VA cells treated with MR-4 did not show significant change, an additional protein band appeared, most likely due to phosphorylation of Bcl-2 (Haldar et al, 1995). Consistent with the increase in the Bax/Bcl-2 mRNA ratio, Bax protein level also increased in WI38VA cells 8$12 \mathrm{~h}$ after MR-4 treatment (Figure 4B). In contrast, the p53, Bcl-2 and Bax level in WI38 cells were barely or much less detectable even after $12 \mathrm{~h}$ of MR-4 treatment (Figure 4B). These results show that MR-4 selectively induced an increase in p53 and Bax/Bcl-2 ratio, consistent with the notion that MR-4 treatment activated 


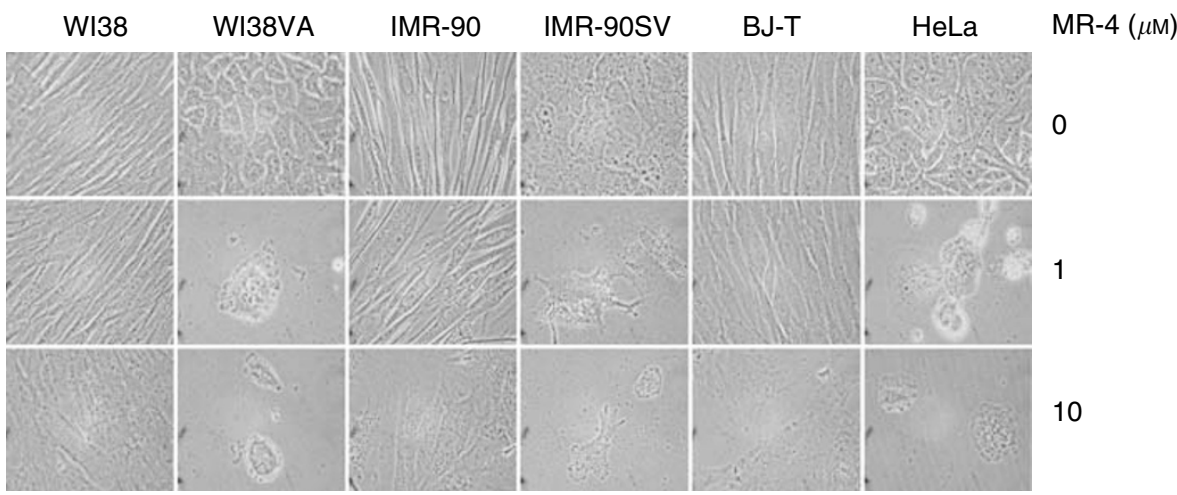

Figure 2 Morphology of various normal and cancer cells after treatment by MR-4. Different normal cell strains including WI38 (normal lung fibroblasts), BJ-T (normal foreskin fibroblasts transfected with telomerase), and IMR-90 (normal fibroblasts) as well as transformed or cancer cell lines including WI38VA (SV40 virally transformed lung fibroblasts), IMR-90SV (SV40 virally transformed fibroblast), and HeLa (cervix cancer) were treated with I or I0 $\mu$ M of MR-4 for 5 days. Morphological analysis was performed by phase contrast light microscopy.

A
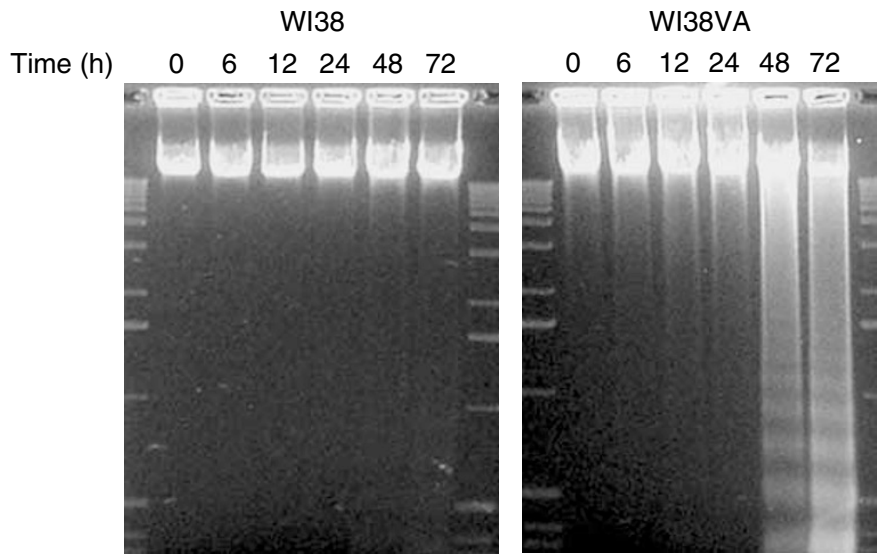

B
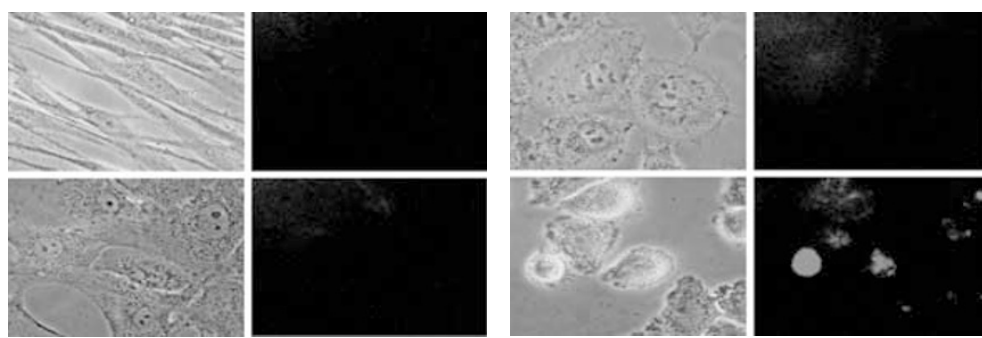

Figure 3 Analysis of apoptotic cell death by $(\mathbf{A})$ DNA fragmentation and (B) caspase activation assay in normal and transformed fibroblasts. Confluent WI38 or WI38VA cultures were treated with MR-4 $(50 \mu \mathrm{M})$ and processed for apoptosis assay. (A) DNA laddering. DNA was isolated from cells at indicated time points and analysed by agarose electrophoresis and ethidium bromide staining. (B) Caspase activation-assay. After $48 \mathrm{~h}$ of MR-4 treatment, the effect on caspase activation was analysed by the CaspACE ${ }^{\mathrm{TM}}$ in situ marker FITC-VAD-FMK, which specifically binds to the activated caspases. After an incubation of $20 \mathrm{~min}$ with the fluorophore, cells were examined by fluorescent microscopy. For each treatment, representative phase contrast (left) and fluorescence (right) micrographs were taken. Upper and lower panels represent controls or MR-4 treated cells, respectively.

mitochondria-mediated apoptotic pathways only in transformed cells.

\section{Mitochondrial morphology and distribution in normal and transformed human fibroblasts}

The upregulation of the Bax/Bcl-2 ratio and $\mathrm{p} 53$ prompted us to analyse possible effect of MR-4 on the mitochondrial morphology and distribution. Cationic fluorophore rhodamine 123 stains intact mitochondria rather specifically (Johnson et al, 1980). We therefore employed rhodamine 123 to monitor possible changes in mitochondrial localisation and clustering in normal and transformed fibroblasts following the MR-4 treatment. We found a drastic change in mitochondrial morphology only in WI38VA cells treated by MR-4. As shown in Figure 5A, MR-4 induced a dose-dependent perinuclear clustering of mitochondria in transformed WI38VA cells, but not in their normal counterpart, WI38 cells. Figure 5B shows the effect of MR-4 on mitochondrial distribution in WI38VA cells at higher magnification. Mitochondria in control WI38VA cells were distributed throughout the cell 

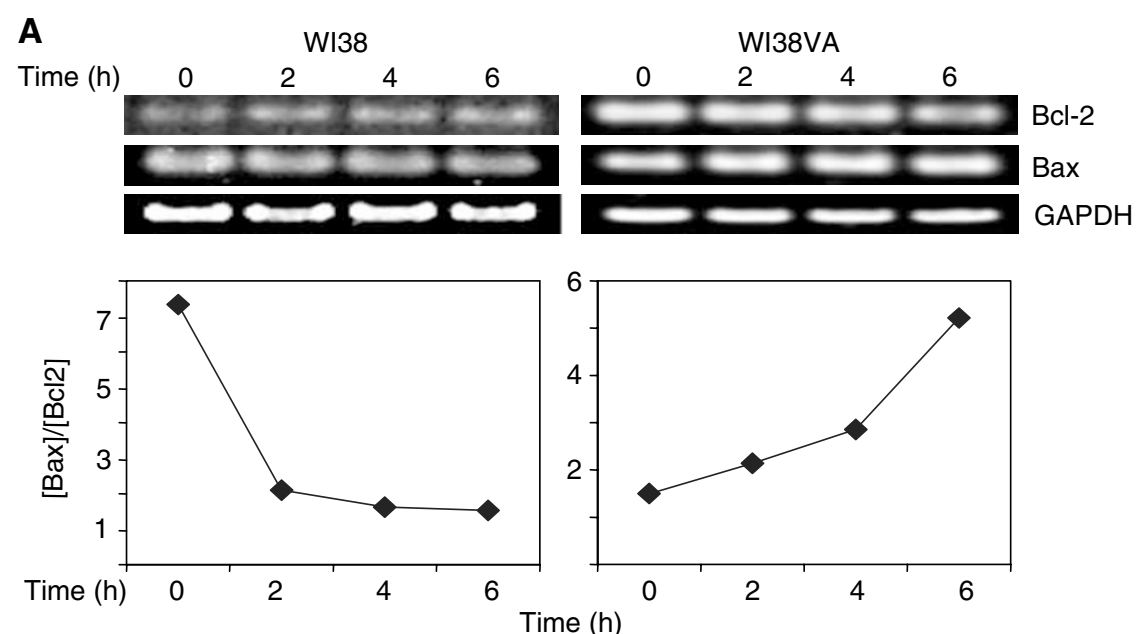

B

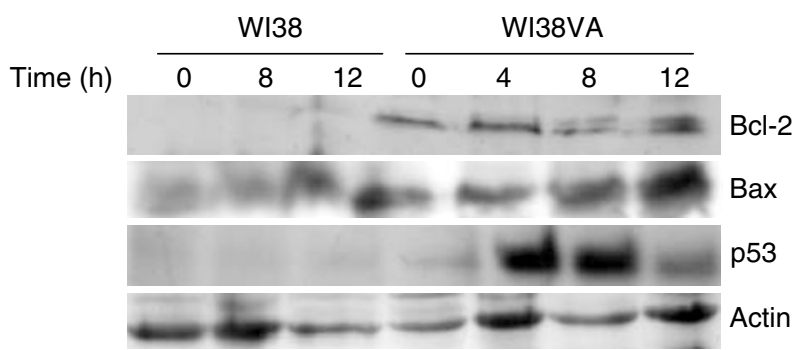

Figure 4 Effect of MR-4 on the expression of apoptotic genes in normal and transformed fibroblasts. (A) RT-PCR analysis. MR-4 (50 $\mu$ M) was applied for $0,2,4$, or $6 \mathrm{~h}$ to WI38 or WI38VA. The induction of Bax and Bcl-2 was analysed by RT-PCR using I $\mu$ g of total RNA. Glyceraldehyde-3-phosphate dehydrogenase (GAPDH) was used as internal standard. Bax/Bcl-2 ratio was determined by densitometry and normalised to GAPDH. (B) Western blot analysis. MR-4 $(50 \mu \mathrm{M})$ was applied for $0,4,8$, or $12 \mathrm{~h}$. Cells were harvested at indicated times and equal amount of cellular protein (30 $\mu \mathrm{g})$ was analysed by SDS-PAGE. Western blot analysis was performed using specific antibodies against Bax, Bcl-2, p53, and actin as described in Materials and Methods.

body, but became aggregated and clustered around the nucleus (perinuclear clustering) after only $3 \mathrm{~h}$ after MR-4 treatment (Figure 5B, bottom $v s$ top). In contrast, the web-like cellular distribution of mitochondria in normal cells was not significantly affected by MR-4 up to $100 \mu \mathrm{M}$. In a time course study using only $5 \mu \mathrm{M}$ MR-4, we observed prominent aggregation of mitochondria in WI38VA cells $6 \mathrm{~h}$ after the treatment (data not shown). When the effect of R-3 was analysed (Figure 5C), we found that R-3 did cause apoptosis and clustering of mitochondria in WI38VA cells, but only at much higher concentrations, up to $500 \mu \mathrm{M}$. Figure $5 \mathrm{D}$ shows the mitochondrial clustering induced by R-3 at $500 \mu \mathrm{M}$ in WI38VA cells. The clustering induced by R-3 was not as prominent and clear as that observed with MR-4 (Figure 5D vs Figure $5 \mathrm{~B}$ ). The effect of MR-4 on mitochondrial clustering was quite dramatic and it was evident even without fluorescent staining (Figure 5B). The observation that MR-4 significantly altered the morphology and distribution of mitochondria in transformed fibroblasts suggests that MR-4 may specifically evoke death signals originating from mitochondria in transformed cells. The fact that R-3 caused apoptosis and mitochondrial clustering only at much higher concentration suggests that MR-4 and R-3 may share a common target site, but the action of MR-4 is more specific and potent.

\section{DISCUSSION}

It has been shown that resveratrol attenuates carcinogenesis or tumour progression both in vitro and in vivo, and is likely the key ingredient for the health beneficial effect of grape wine (Jeandet et al, 1991; Jang et al, 1997; Elattar and Virji, 1999; Hsieh and $\mathrm{Wu}$, 1999; Lu and Serrero, 1999; Surh et al, 1999; Tsan et al, 2000;
Gusman et al, 2001; Ding and Adrian, 2002; Narayanan et al, 2003; Zhou et al, 2003). Although the antiproliferative effect of resveratrol seems to be due to the induction of apoptosis (Hsieh and Wu, 1999; Surh et al, 1999; Tsan et al, 2000; Ding and Adrian, 2002; Zhou et al, 2003), current research on the use of resveratrol as potential cancer chemoprevention agent has been largely limited to the study with only cancer cells. In an attempt to determine whether chemical modification of the stilbene backbone of resveratrol may further enhance its biological activity and differentiate cancer cells from their normal counterparts, we have synthesised and tested a number of hydroxy- and methoxystilbenes (Lu et al, 2001). Among them, MR-4 is by far not only the most potent one but also is the one that exhibits the most striking differential growth inhibitory effect against cancer cells (Figures 1 and 2). As previously noted (Chen et al, 1998; Lu et al, 2001; Gosslau and Chen, 2004), when tested as a potential anticancer agent it is desirable that the compound has minimal detrimental effect on the growth or survival of normal cells. In this regard, MR-4 may be superior to natural resveratrol as a candidate for chemoprevention agent. Nevertheless, further work on toxicity is needed to establish this possibility. Since MR-4 did not exhibit any antioxidant activity as demonstrated by the thiobarbituric acid assay (A Gosslau and KY Chen, unpublished result), the enhanced anticancer activity of MR-4 does not appear to be related to antioxidation, as known to be associated with various polyphenol compounds. The fact that R-4 and MR-4, but not R-3 or MR-3 (the trimethoxy derivative of R-3), exhibits differential growth inhibitory effect (Lu et al, 2001 and Figures 1 and 2) suggests that structure-related mechanisms (an additional hydroxyl or methoxyl group) may be crucial in revealing the target sites that differentiate cancer cells from their normal counterparts. On the other hand, we 
A
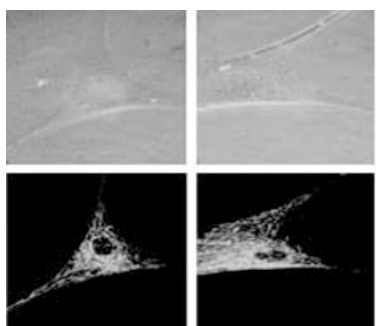

WI38

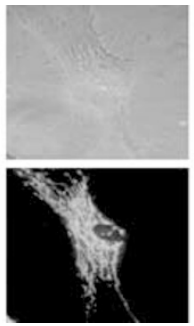

WI38VA
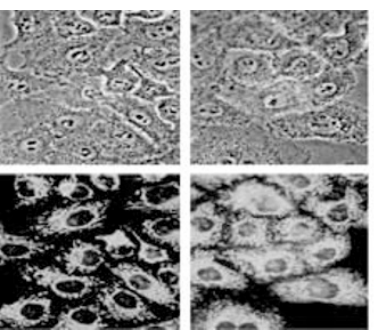

25

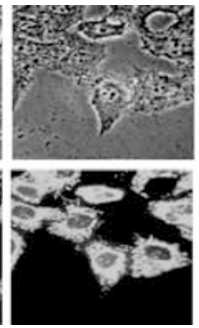

50

WI38VA
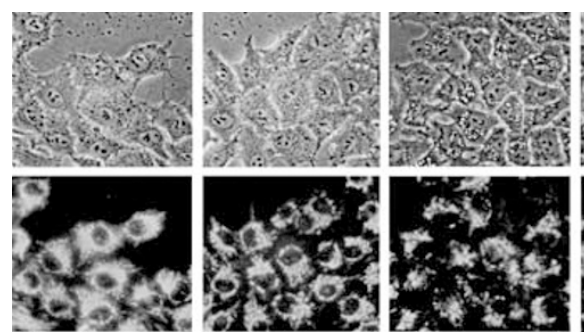

R-3

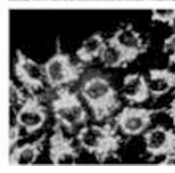

100

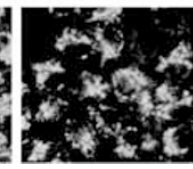

250
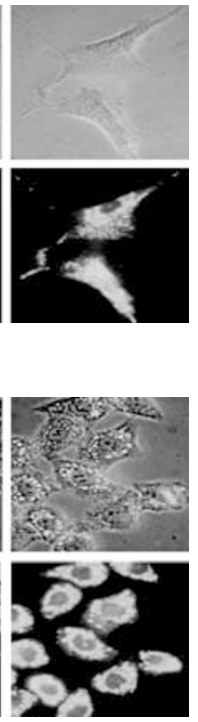

100
B WI38VA

(Control)

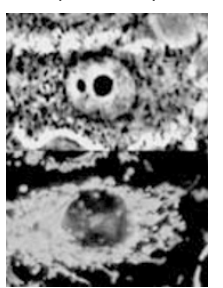

WI38VA

(MR-4, 100 $\mu \mathrm{M}$ )

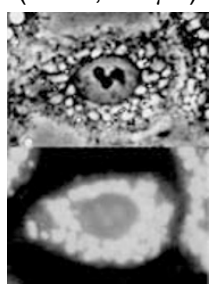

D WI38VA (R-3, 500 $\mu \mathrm{m})$

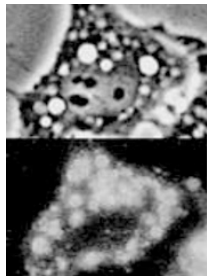

Figure 5 Effect of MR-4 and R-3 on the mitochondrial morphology in normal and transformed fibroblasts. (A) Dose-response study of the effect of MR-4 on mitochondrial localisation in WI38 and WI38VA cells. Cells were treated with different concentrations of MR-4 for $3 \mathrm{~h}$ and then stained with rhodamine 123 for $20 \mathrm{~min}$. Cells were then analysed either by phase contrast (top of each panel) or fluorescence microscopy (bottom of each panel) at a final magnification of $\times 1000$. (B) Effect of MR-4 on WI38VA cells. Cells treated without (control) or with MR-4 (I00 $\mu$ M) for $3 \mathrm{~h}$ and analysed at a magnification of $\times 5000$. (C) Dose-response study of the effect of R-3 on mitochondrial localisation in WI38VA cells. Cells were treated with R-3 at indicated concentrations for $3 \mathrm{~h}$ and then stained with rhodamine 123 for $20 \mathrm{~min}$ and then analysed by phase contrast (top panels) and fluorescent (bottom panels) microscopic examination. (D) Effect of R-3 on WI38VA cells. Cells were treated with R-3 at $500 \mu \mathrm{M}$ for $3 \mathrm{~h}$ and then examined under phase contrast (upper) and fluorescent (bottom) microscopy at a magnification of $\times 5000$.

cannot rule out the possibility that the differences between the effects of R-3 and MR-4 reflect differences in the uptake of these compounds.

The close correlation between the growth inhibition (Figures 1 and 2) and the effect of MR-4 on apoptosis in transformed cells, as determined by morphological damage, DNA laddering and caspase activation (Figure 3), suggest that the target sites of MR-4 could be any components involved in apoptotic pathway or the upstream events that lead to apoptosis. The tumour suppressor protein p53 is known to function as a gatekeeper controlling critical cell cycle checkpoints (Levine, 1997) and also act as a key player in mediating apoptosis through modulating the Bax to Bcl-2 ratio and the regulation of mitochondrial ROS generation (Miyashita and Reed, 1995; Johnson et al, 1996; Levine, 1997; Yin et al, 1997; Sheikh and Fornace, 2000; Pietenpol and Stewart, 2002). The findings that MR-4 led to the induction of p53, increases in Bax and $\mathrm{Bax} / \mathrm{Bcl}-2$ ratio (Figure 4) and caused a rapid perinuclear clustering of mitochondria (Figure 5) in transformed cells, but not in normal cells, could explain the selective apoptotic effect of MR-4 in cancer cells. The appearance of Bcl-2 doublet in MR-4 treated WI38VA cells (Figure 4B) also suggests that the phosphorylation of Bcl-2 may contribute to the apoptotic process in transformed cells, as it has been shown that $\mathrm{Bcl}-2$ phosphorylation not only inactivates itself but also facilitates permeabilisation of mitochondria membrane (Haldar et al, 1995).

In accordance with the notion that MR-4 may trigger the mitochondria-mediated apoptosis in transformed cells, we observed prominent condensation and clustering of mitochondria around the nucleus in cancer cells, but not in normal cells after MR-4 treatment (Figure 5A and B). This dramatic morphological change becomes obvious within $3 \mathrm{~h}$ after treatment and represents one of the earlier events induced by MR-4. Our observation that R-3 also induced clustering of mitochondria, although at much higher concentrations as compared to MR-4 (Figure 5C and D), suggests that MR-4 and R-3 may share a common target site. Other investigators have reported that mitochondria-mediated apoptosis sometimes is accompanied with morphological changes and redistribution of mitochondria in apoptotic cells (Desagher and 
Martinou, 2000). The mechanism of clustering is unknown. The possible causal relationship between mitochondria clustering and apoptosis needs to be clarified. One possible scenario is that MR-4 causes mitochondrial clustering and depolarisation in cancer cells, which results in the release of proapoptotic factors and triggers apoptosis. We observed the formation of crystal-like structures specifically around mitochondria of transformed fibroblasts treated by MR-4 (Figure 5B). The identification of the nature of these structures may help to resolve the apoptotic mechanisms induced by MR- 4 in cancer cells. In addition, other targets of MR-4 particularly can be further investigated by microarray technology.

Taken together, our data show that the resveratrol analogue MR-4 exhibits potent and selective growth inhibitory effect to cancer cells. The specific induction of perinuclear clustering of mitochondria and the activation of the mitochondria-mediated apoptotic pathway seem to be the major cause for the striking differential inhibitory effect of MR-4. Identification of the target of MR-4 in cancer cells will be the key for our understanding of the action of MR-4. A major challenge in cancer therapy is to search or synthesise drugs that kill tumour cells while preserve normal cells. In this regard, polyhydroxystibene may serve as a useful chemical platform for developing more potent and selective anticancer reagents.

\section{ACKNOWLEDGEMENTS}

This work was supported by the Commission on Science and Technology, State of New Jersey as a component of the Pioneering Nutraceutical Research Program. We also acknowledge the helpful discussions with Drs Geetha Ghai, Robert Rosen, and David Evans.

\section{REFERENCES}

Aziz M, Kumar R, Ahmad N (2003) Cancer chemoprevention by resveratrol: in vitro and in vivo studies and the underlying mechanisms (review). Int J Oncol 23: 17-28

Bachelor F, Loman A, Snowdon L (1970) Synthesis of pinosylvin and related heartwood stilbenes. Can J Chem 48: 1554-1557

Berg K, Hansen M, Nielsen S (1990) A new sensitive bioassay for precise quantification of interferon activity as measured via the mitochondrial dehydrogenase function in cells (MTT-method). APMIS 98: 156-162

Bernhard D, Schwaiger W, Crazzolara R, Tinhofer I, Kofler R, Csordas A (2003) Enhanced MTT-reducing activity under growth inhibition by resveratrol in CEM-C7H2 lymphocytic leukemia cells. Cancer Lett 195: $193-199$

Bhat K, Pezzuto J (2002) Cancer chemopreventive activity of resveratrol. Ann NY Acad Sci 957: 210-229

Budihardjo I, Oliver H, Lutter M, Luo X, Wang X (1999) Biochemical pathways of caspase activation during apoptosis. Annu Rev Cell Dev Biol 15: $269-290$

Chen Z, Schell J, Ho C, Chen K (1998) Green tea epigallocatechin gallate shows a pronounced growth inhibitory effect on cancerous cells but not on their normal counterparts. Cancer Lett 129: 173 - 179

Compton M (1992) A biochemical hallmark of apoptosis: internucleosomal degradation of the genome. Cancer Metastasis Rev 11: 105-119

Davies K (2000) Oxidative stress, antioxidant defenses, and damage removal, repair, and replacement systems. IUBMB Life 50: 279-289

Desagher S, Martinou J (2000) Mitochondria as the central control point of apoptosis. Trends Cell Biol 10: 369-377

Ding X, Adrian T (2002) Resveratrol inhibits proliferation and induces apoptosis in human pancreatic cancer cells. Pancreas 25: e71-e76

Drewes S, Fletcher L (1974) Polyhydroxystilbenes from the heartwood of Schotia brachypetala. J Chem Soc Perkin Trans 1: 961-962

Elattar T, Virji A (1999) The effect of red wine and its components on growth and proliferation of human oral squamous carcinoma cells. Anticancer Res 19: $5407-5414$

Gosslau A, Chen KY (2004) Nutraceuticals, apoptosis, and disease prevention. Nutrition 20: $95-102$

Green D, Reed J (1998) Mitochondria and apoptosis. Science 281: 1309 1312

Gusman J, Malonne H, Atassi G (2001) A reappraisal of the potential chemopreventive and chemotherapeutic properties of resveratrol. Carcinogenesis 22: 1111-1117

Haldar S, Jena N, Croce C (1995) Inactivation of Bcl-2 by phosphorylation. Proc Natl Acad Sci USA 92: 4507-4511

Hengartner M (2000) The biochemistry of apoptosis. Nature 407: 770-776

Hsieh T, Wu J (1999) Differential effects on growth, cell cycle arrest, and induction of apoptosis by resveratrol in human prostate cancer cell lines. Exp Cell Res 249: 109-115

Huang C, Ma W, Goranson A, Dong Z (1999) Resveratrol suppresses cell transformation and induces apoptosis through a p53-dependent pathway. Carcinogenesis 20: $237-242$

Hung L, Chen J, Lee R, Liang H, Su M (2001) Beneficial effects of astringinin, a resveratrol analogue, on the ischemia and reperfusion damage in rat heart. Free Radical Biol Med 30: $877-883$
Igney F, Krammer P (2002) Death and anti-death: tumour resistance to apoptosis. Nat Rev Cancer 2: 277-288

Jang M, Cai L, Udeani G, Slowing K, Thomas C, Beecher C, Fong H, Farnsworth N, Kinghorn A, Mehta R, Moon R, Pezzuto J (1997) Cancer chemopreventive activity of resveratrol, a natural product derived from grapes. Science 275: 218-220

Jeandet P, Bessis R, Gautheron B (1991) The production of resveratrol by grape berries in different developmental stages. Am J Enol Viticult 42: 41

Johnson L, Walsh M, Chen L (1980) Localization of mitochondria in living cells with rhodamine 123. Proc Natl Acad Sci USA 77: 990-994

Johnson T, Yu Z, Ferrans V, Lowenstein R, Finkel T (1996) Reactive oxygen species are downstream mediators of p53-dependent apoptosis. Proc Natl Acad Sci USA 93: 11848-11852

Korsmeyer S (1999) BCL-2 gene family and the regulation of programmed cell death. Cancer Res 59: $1693 \mathrm{~s}-1700 \mathrm{~s}$

Korsmeyer S, Shutter J, Veis D, Merry D, Oltvai Z (1993) Bcl-2/Bax: a rheostat that regulates an anti-oxidant pathway and cell death. Semin Cancer Biol 4: 327-332

Levine A (1997) p53, the cellular gatekeeper for growth and division. Cell Mol Biol 88: 323-331

Lu J, Ho C, Ghai G, Chen K (2001) Resveratrol analog, 3,4,5,4'tetrahydroxystilbene, differentially induces pro-apoptotic p53/Bax gene expression and inhibits the growth of transformed cells but not their normal counterparts. Carcinogenesis 22: 321-328

$\mathrm{Lu}$ R, Serrero G (1999) Resveratrol, a natural product derived from grape, exhibits antiestrogenic activity and inhibits the of human breast cancer cells. Cell Physiol 179: 297-304

Miyashita T, Reed J (1995) Tumor suppressor p53 is a direct transcriptional activator of the human bax gene. Cell Mol Biol 80: 293-299

Narayanan B, Narayanan N, Re G, Nixon D (2003) Differential expression of genes induced by resveratrol in LNCaP cells: P53-mediated molecular targets. Int J Cancer 104: 204-212

Peter M, Krammer P (2003) The CD95(APO-1/Fas) DISC and beyond. Cell Death Differ 10: $26-35$

Pietenpol J, Stewart Z (2002) Cell cycle checkpoint signaling: cell cycle arrest versus apoptosis. Toxicology 181-182: 475-481

Reed J (2002) Apoptosis-based therapies. Nat Rev Drug Discov 1: 111-121

Reed J (2003) Apoptosis-targeted therapies for cancer. Cancer Cell 3: $17-22$

Savouret J, Quesne M (2002) Resveratrol and cancer: a review. Biomed Pharmacother 56: $84-87$

Sheikh M, Fornace AJ (2000) Role of p53 family members in apoptosis. J Cell Physiol 182: 171-181

Strater J, Moller P (2000) Expression and function of death receptors and their natural ligands in the intestine. Ann NY Acad Sci 915: $162-170$

Surh Y, Hurh Y, Kang J, Lee E, Kong G, Lee S (1999) Resveratrol, an antioxidant present in red wine, induces apoptosis in human promyelocytic leukemia (HL-60) cells. Cancer Lett 140: 1-10

Susin S, Zamzami N, Castedo M, Daugas E, Wang H, Geley S, Fassy F, Reed J, Kroemer G (1997) The central executioner of apoptosis: multiple connections between protease activation and mitochondria 
in Fas/APO-1/CD95- and ceramide-induced apoptosis. J Exp Med 186: $25-37$

Tsan M, White J, Maheshwari J, Bremner T, Sacco J (2000) Resveratrol induces Fas signalling-independent apoptosis in THP-1 human monocytic leukaemia cells. Br J Haematol 109: 405-412

vanGurp M, Festjens N, Loo Gv, Saelens X, Vandenabeele P (2003) Mitochondrial intermembrane proteins in cell death. Biochem Biophys Res Commun 304: 487-497
Wu J, Wang Z, Hsieh T, Bruder J, Zou J, Huang Y (2001) Mechanism of cardioprotection by resveratrol, a phenolic antioxidant present in red wine (Review). Int J Mol Med 8: 3-17

Yin C, Knudson C, Korsmeyer S, Dyke TV (1997) Bax suppresses tumorigenesis and stimulates apoptosis in vivo. Nature 385: 637-640

Zhou H, Yan Y, Sun Y, Zhu J (2003) Resveratrol induces apoptosis in human esophageal carcinoma cells. World J Gastroenterol 9: $408-411$ 